\title{
Электронные состояния атомов в монослоях, адсорбированных на карбиде кремния
}

\author{
(C) С.Ю. Давыдов ${ }^{1}$, О.В. Посредник ${ }^{2}$ \\ ${ }^{1}$ Физико-технический институт им. А.Ф. Иоффре Российской академии наук, \\ 191021 Санкт-Петербург, Россия \\ ${ }^{2}$ Санкт-Петербургский государственный электротехнический университет (ЛЭТИ), \\ 197376 Санкт-Петербург, Россия \\ E-mail: Sergei_Davydov@mail.ru
}

Поступила в Редакцию 12 октября 2021 г.

В окончательной редакции 18 октября 2021 г.

Принята к публикации 18 октября 2021 г.

С учетом диполь-дипольного отталкивания и обменных взаимодействий между адатомами приведены оценки перехода заряда между монослоем ад-сорбата и $\mathrm{SiC}$-подложкой. Продемонстрировано, что все виды взаимодействия ведут к деполяризации адсорбированного слоя (адслоя). Проанализирована роль зонных и локальных состояний адатомов. Предложены способы экспериментальной проверки полученных результатов.

Ключевые слова: диполь-дипольное взаимодействие, прямой и косвенный обмены.

DOI: 10.21883/FTP.2022.02.51965.9751

\section{1. Введение}

Несмотря на длительную историю изучения, карбид кремния, характеризуемый большим количеством политипов с существенно различающимися запрещенными зонами и высокой стойкостью к агрессивным средам, попрежнему остается привлекательным объектом исследований [1-4]. Следует подчеркнуть, однако, что адсорбционная способность политипов $\mathrm{SiC}$ изучена сравнительно слабо, несмотря на важность этой характеристики как с фундаментальной, так и с технологической точек зрения. В предыдущих работах нами была рассмотрена адсорбция одиночных атомов I, VII [5] и II, VI [6] групп на C- и $\mathrm{Si-гранях} \mathrm{кубического} \mathrm{и} \mathrm{основных} \mathrm{гексагональных}$ политипов карбида кремния. Здесь мы обратимся к конечным концентрациям адатомов, т. е. к адсорбированным слоям (адслоям). При этом удобно ввести степень покрытия $\Theta=N / N_{\mathrm{ML}}$, где $N\left(N_{\mathrm{ML}}\right)-$ концентрация адсорбированных частиц в слое (монослое).

Задача об адсорбции при конечной концентрации адатомов предполагает учет взаимодействия между ними. Существуют три основных канала такого взаимодействия [7]. Если между слоем и подложкой имеет место переход заряда, то между адатомами возникает дипольдипольное взаимодействие. Двумя другими каналами являются обменные взаимодействия: косвенный обмен адатомов через электронные состояния подложки и прямой обмен, возникающий при перекрытии орбиталей соседних адатомов. Так как при адсорбции одиночных атомов I, II, VI и VII групп на SiC имеет место существенный переход заряда $[5,6]$, начнем именно с рассмотрения диполь-дипольного взаимодействия. В дальнейшем будем считать, что все адатомы одинаковы и занимают эквивалентные позиции на поверхности подложки, так что в адслое действует диполь-дипольное отталкивание.

\section{2. Диполь-дипольное отталкивание}

Влияние диполь-дипольного взаимодействия на электронное состояние атомов в слоях на полупроводнике изучалось нами в работе [8]. В соответствии с результатами работ $[5,6]$ число заполнения адатома $n=n_{b}+n_{l}$, где $n_{b}$ - зонный вклад, который при нулевой температуре равен вкладу валентной зоны $n_{v}, n_{l}$ - вклад локальных состояний с энергиями $\omega_{l}$, лежащими в запрещенной зоне подложки. Как показано при нулевой температуре в работе [8] числа заполнения в адслое равны:

$$
\begin{gathered}
n_{v}(\Theta)=\frac{1}{\pi} \operatorname{arcctg} \frac{\varepsilon_{a}(\Theta)+R}{\Gamma}, \quad R=\frac{E_{g}}{2} \sqrt{1+\frac{4 \Gamma}{\pi E_{g}}}, \\
n_{l}(\Theta)=v_{l}(\Theta) \cdot \Theta_{H}\left(E_{\mathrm{F}}-\omega_{l}(\Theta)\right) \\
v_{l}(\Theta)=\left(1+\frac{\Gamma}{\pi} \frac{E_{g}}{\left(E_{g} / 2\right)^{2}-\omega_{l}^{2}(\Theta)}\right)^{-1} .
\end{gathered}
$$

Здесь $E_{g}-$ ширина запрещенной зоны подложки, $\Gamma$ - полуширина квазиуровня изолированного адатома, $\varepsilon_{a}(\Theta)=\varepsilon_{a}-\varepsilon_{d i p}(\Theta), \varepsilon_{d i p}(\Theta)=\Theta^{3 / 2} \xi Z_{a}(\Theta), \varepsilon_{a}-$ энергия уровня изолированного адатома, $\xi=2 e^{2} d^{2} N_{\mathrm{ML}}^{3 / 2} A-$ константа дипольного взаимодействия, $e-$ элементарный заряд, $d-$ длина адсорбционной связи, $A \sim 10-$ множитель, слабо зависящий от геометрии адслоя, $\Theta_{\mathrm{H}}(\ldots)$ - функция Хэвисайда, $Z(\Theta)$ - заряд адатома в адслое, равный $1-n(\Theta)$, если уровень адатома изначально (до адсорбции) заполнен (адатомыдоноры), и $-n(\Theta)$, если уровень адатома изначально пуст (адатомы-акцепторы). Общий анализ зависимостей (1) и (2) проведен в работе [8]. Здесь мы обратимся к численным оценкам значений $n_{\mathrm{ML}}=n(\Theta=1)$ и $Z_{\mathrm{ML}}=Z(\Theta=1)$ для конкретных адсорбционных систем. 
В качестве адсорбатов рассмотрим атомы $\mathrm{Na}$ и $\mathrm{Cl}$, являющиеся типичными донором и акцептором. При адсорбции на $\mathrm{Si}$ - или С-грани карбида кремния площадь, приходящаяся на один атом, равна $S=3 \sqrt{3} a^{2} / 4$, где $a$ - расстояние между ближайшими соседними адатомами. Поскольку $N_{\mathrm{ML}}=1 / S^{1}$, полагая для простоты $a=2 \AA$, получим $N_{\mathrm{ML}} \approx 17.5 \cdot 10^{-2} \AA^{-2}$. Так как для С-грани $d_{\mathrm{Na}}=2.64$ и $d_{\mathrm{Cl}}=1.76 \AA[5,9,10]$, получаем $\xi_{\mathrm{Na}} \approx 100$ и $\xi_{\mathrm{Cl}} \approx 50$ эВ. Для $\mathrm{Si}$-грани $d_{\mathrm{Na}}=3.05$, $d_{\mathrm{Cl}}=2.17 \AA$ и $\xi_{\mathrm{Na}} \approx 140, \xi_{\mathrm{Cl}} \approx 70$ эВ. В соответствии с результатами работы [5] имеем $\Gamma=0.63(3.03)$ и 0.35(1.31) эВ для C- и Si-граней при адсорбции натрия (хлора). Если подложкой является политип $6 H$-SiC $\left(E_{g}=3\right.$ эВ $) \quad p$-типа проводимости, то при адсорбции натрия на С-грани имеем $\varepsilon_{a}=1.14$ эВ, $\omega_{l}=0.86$ эВ, $Z_{0}=Z(\Theta=0)=Z^{>}=0.93$ (символ $>(<)$ означает, что локальные уровни лежат выше (ниже) уровня Ферми и поэтому являются пустыми (заполненными)); при адсорбции на $\mathrm{Si}$-грани имеем $\varepsilon_{a}=1.00$ эВ, $\omega_{l}=0.86$ эВ, $Z_{0}=0.96$ (энергия отсчитывается от центра запрещенной зоны) [5]. При адсорбции хлора на С-грани $\varepsilon_{a}=-0.72$ эВ, $\omega_{l}=-0.32$ эВ, $Z_{0}=Z(\Theta=0)=Z^{<}=-0.78$; при адсорбции на Si-грани имеем $\varepsilon_{a}=-0.33$ эВ $\omega_{l}=-0.21$ эВ, $Z_{0}=-0.86[5]^{2}$, где $6 H$-SiC имеет $n$-тип проводимости. Здесь тип допирования выбирался из-за больших значений $\left|Z_{0}\right|$ для наглядности демонстрации деполяризации адатомов при росте $\Theta$. При адсорбции на металлах и полупроводниках зависимость $|Z(\Theta)|$ является либо плавно убывающей функцией (при этом убывает и наклон $|\partial Z(\Theta) / \partial \Theta|)$, либо функцией со сравнительно слабым минимумом при промежуточных покрытиях $\Theta^{*} \sim 0.5[7,11]$. В дальнейшем ограничимся оценками $n_{\mathrm{ML}}=n(\Theta=1)$ и $Z_{\mathrm{ML}}=Z(\Theta=1)$.

Так как константа дипольного взаимодействия $\xi$ является самой большой энергетической величиной задачи, предположим, что $\xi\left|Z_{\mathrm{ML}}\right| \gg\left|\varepsilon_{a}+R\right|, \Gamma$. Для натрия из (1) и (2) находим соответственно

$$
n_{v}^{\mathrm{ML}} \sim 1-\Gamma / \pi \xi Z_{\mathrm{ML}}, \quad n_{l}=0 .
$$

Так как $v_{l}^{\mathrm{ML}} \sim 0$, получаем $Z_{\mathrm{ML}} \sim \sqrt{\Gamma / \pi \xi} \sim 0.04$ при адсорбции на $\mathrm{C}$ - и $\mathrm{Si}$-грани $6 H-\mathrm{SiC}$-типа. Таким образом, имеет место сильная деполяризация, в результате

\footnotetext{
${ }^{1}$ Необходимо отметить, что $N_{\mathrm{ML}}$ и $\Theta$ являются плохо определенными параметрами. Поэтому экспериментаторы уже практически отказались от их использования и предпочитают говорить о дозе облучения поверхности подложки атомами. В теориях из первых принципов рассматривают различные геометрии адслоя, при этом полагают, что его структура соизмерима со структурой подложки. При модельном подходе часто принимают $N_{\mathrm{ML}} \sim 1 / \pi r_{a}^{2}$, где $r_{a}-$ атомный радиус адсорбата. Подобная оценка справедлива и для несоизмеримых структур адслоя и грани подложки.

2 Все заряды адатомов II и VI групп по определению отрицательны. В таблицах работ $[5,6]$ для этих групп приведены $\left|Z^{>(<)}\right|$, а не $Z^{>}(<)$как в тексте. Исключением являются данные по адсорбции галогенов на $\mathrm{Si}$-грани $3 \mathrm{C}-\mathrm{SiC}[5]$, где приведены именно значения $Z^{>(<)}$. Следует также учесть, что в формулах (5) работ $[5,6]$ в знаменателях множителя перед скобками должно стоят $r_{a}^{2}$, а не $d^{2}$.
}

которой адсорбированный ион $\mathrm{Na}$ теряет практически весь свой заряд. Очевидно, что тот же результат будет иметь место и при адсорбции натрия на политипе $p-4 H-\mathrm{SiC}\left(E_{g}=3.23\right.$ эВ). Более того, порядковые оценки (3) и вывод о сильной деполяризации можно применить и к другим щелочным и щелочно-земельным металлам (см. работы $[5,6])$.

Перейдем теперь к адсорбции хлора на С- и Si-гранях $6 H$-SiC $n$-типа. Вновь предполагая, что $\xi\left|Z_{\mathrm{ML}}\right| \gg\left|\varepsilon_{a}+R\right|, \Gamma$, получим

$$
\begin{gathered}
n_{v}^{\mathrm{ML}} \sim-\Gamma / \pi \xi Z_{\mathrm{ML}}, \\
v_{l}^{\mathrm{ML}} \sim\left(2 \pi E_{g} / \Gamma\right) \exp \left(-\pi \xi Z_{\mathrm{ML}} / \Gamma\right) .
\end{gathered}
$$

Отсюда $\quad n_{v}^{\mathrm{ML}} \sim-2 \cdot 10^{-2} / Z_{\mathrm{ML}}, \quad n_{l}^{\mathrm{ML}} \sim 0.8 \cdot 10^{-2} \quad$ и $Z_{\mathrm{ML}} \sim-\sqrt{\Gamma / \pi \xi} \sim-0.15 \sim 0.15$ (С-грань) и $n_{v}^{\mathrm{ML}} \sim$ $\sim-6 \cdot 10^{-3} / Z_{\mathrm{ML}}, n_{l}^{\mathrm{ML}} \sim 0$, так что $Z_{\mathrm{ML}} \sim-\sqrt{\Gamma / \pi \xi} \sim$ $\sim 0.08$ (Si-грань). Таким образом, имеет место существенная деполяризация, причем, как и в случае щелочных металлов, заряд атомов монослоя определяется превалирующим вкладом валентной зоны. Сделанные порядковые оценки могут быть распространены на подложку $4 H$-SiC $p$-типа и на адсорбцию стронция и бария. Отметим также, что качественные выводы о деполяризации адатомов не зависят от типа проводимости подложки.

Энергия связи адатома с подложкой (энергия адсорбции) при $\Theta=1$ равна $E_{\mathrm{ads}}^{\mathrm{ML}}=E_{\mathrm{ion}}^{\mathrm{ML}}+E_{\mathrm{met}}^{\mathrm{ML}}$, где оценки величин ионной и металлической составляющих равны соответственно

$$
E_{\mathrm{ion}}^{\mathrm{ML}}=-\frac{\left(Z_{\mathrm{ML}} e\right)^{2}}{4 d}, \quad E_{\mathrm{met}}^{\mathrm{ML}}=-\frac{\hbar^{2}}{2 m r_{a}^{2}}\left(1-\frac{r_{a}^{2}}{d^{2}}+\frac{r_{a}^{2}}{a_{0}^{2}}\right) .
$$

В (5) для $E_{\text {met }}^{\mathrm{ML}}$ учтено, что в отличие от адсорбции одиночного атома, где электрон может перейти лишь в объем подложки, при $\Theta=1$ возможна дополнительная делокализация электронов в плоскости адслоя. При переходе от адсорбции одиночного атома к монослою изменение энергии адсорбции равно $\delta E_{\text {ada }}=\delta E_{\text {ion }}+\delta E_{\text {met }}$, где $\delta E_{\text {ion }}=e^{2}\left(Z_{0}^{2}-Z_{\mathrm{ML}}^{2}\right) / 4 d^{2}$ и $\delta E_{\text {met }}=-\hbar^{2} / 2 m d^{2}$. Введем отношение $\beta=-\delta E_{\text {ion }} / \delta E_{\text {met }} \sim d\left(Z_{0}^{2}-Z_{\mathrm{ML}}^{2}\right)$, где $d$ измеряется в $\AA$. При $\beta<1$ энергия связи $E_{b}=-E_{\mathrm{ads}}$ адатома с подложкой в монослое больше, чем у одиночного атома. Для адсорбции $\mathrm{Na}$ на $p-6 H-\mathrm{SiC}$ имеем $\beta \sim 2$

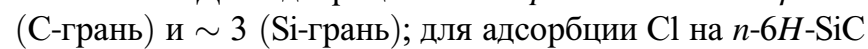
имеем $\beta \sim 1$ (С-грань) и $\sim 1.50$ (Si-грань).

\section{3. Обменные взаимодействия}

При высоких (близких к монослойному) покрытиях, когда возникает перекрытие электронных оболочек соседних адатомов, электрон получает возможность переходить с адатома на адатом [7]. Такая межатомная связь называется прямым, или кинетическим обменом. Как и в предыдущем разделе, рассмотрим монослой адатомов, расположенных в tор-позициях и образующих гексагональную двумерную (2D) решетку на гранях (0001) 
и $(000 \overline{1})$ карбида кремния. В рамках адсорбционного подхода и низкоэнергетического приближения плотность состояний такой решетки дается выражением (10) из работы [12]. Выражение это, однако, достаточно громоздкое и получить с его помощью число заполнения адатома решетки в аналитическом виде возможным не представляется. Прибегнем поэтому к упрощениям.

Воспользовавшись выражением (6) из работы [13], функцию Грина для адатома эпитаксиальной гексагональной 2D решетки можно записать в виде

$$
G(\omega, \mathbf{q})=g_{a}(\omega)\left[1-g_{a}^{2}(\omega) t^{2} f^{2}(\mathbf{q})\right]^{-1} .
$$

Здесь $f(\mathbf{q})=3|\mathbf{q}| a_{0} / 2$ (низкоэнергетическое приближение), q - 2D волновой вектор, отсчитываемый от точки К зоны Бриллюэна 2D гексагональной решетки, $\omega-$ энергетическая переменная, $t-$ энергия перескока электрона между ближайшими соседями в решетке, $g_{a}(\omega)=\left(\omega-\varepsilon_{a}-\Lambda(\omega)+i \Gamma(\omega)\right)^{-1}-$ функция Грина одиночного адатома, $\Lambda(\omega)$ и $\Gamma(\omega)$ - функции сдвига и уширения атомного уровня $\varepsilon_{a}$. Как и в $[5,6]$, для описания плотности состояний подложки $\rho_{\text {sub }}(\omega)$ будем использовать модель Халдейна-Андерсона, согласно которой $\rho_{\text {sub }}(\omega)=\rho_{s}$ для $|\omega| \geq E_{g} / 2$ и $\rho_{\text {sub }}(\omega)=0$ при $|\omega|<E_{g} / 2$, где нуль энергии помещен в центр запрещенной зоны. Тогда $\Gamma \equiv \pi V^{2} \rho_{s}=$ const и $\Lambda(\omega)=(\Gamma / \pi) \ln \left|\left(\omega-E_{g} / 2\right) /\left(\omega+E_{g} / 2\right)\right| \quad$ и $\quad \Gamma \equiv \pi V^{2} \rho_{s}$ $=$ const, где $V$ - матричный элемент взаимодействия подложка-адатом. Так как максимальное значение $|\mathbf{q}|$ равно вектору обрезания $q_{c}=2 \sqrt{2 \pi} / a_{0} \sqrt{3 \sqrt{3}}$ $\approx 2.2 / a_{0}[14]$, максимальное значение функции $f(\mathbf{q})$ равно $f_{\text {max }}=f\left(q_{c}\right)=\sqrt{2 \pi / \sqrt{3}} \approx 1.90$, среднее значение $\bar{f}=f\left(q_{c} / 2\right) \approx 0.95$. С другой стороны, функция Грина адсорбированного димера равна (см. [15])

$$
G_{\operatorname{dim}}(\omega)=g_{a}(\omega)\left[1-g_{a}^{2}(\omega) t^{2}\right]^{-1},
$$

так что $G(\omega)=G\left(\omega, q_{c} / 2\right) \approx G_{\operatorname{dim}}(\omega)$. Таким образом, в целях упрощения последующих оценок (например, чисел заполнения) эпитаксиальный слой может быть моделирован димером.

Функции Грина димера соответствует плотность состояний

$$
\begin{gathered}
\tilde{\rho}(\omega)=\frac{1}{2}\left[\rho_{-}(\omega)+\rho_{+}(\omega)\right], \\
\rho_{ \pm}(\omega)=\frac{1}{\pi} \frac{\Gamma(\omega)}{\left(\omega-\varepsilon_{a}-\Lambda(\omega)\right)^{2}+\Gamma^{2}(\omega)} .
\end{gathered}
$$

Здесь и в дальнейшем тильдой отмечаются все величины, относящиеся к решетке. Тогда можно показать, что $\widetilde{n}_{v(l)}=\left(n_{v(l)}^{-}+n_{v(l)}^{+}\right) / 2$, где

$$
\begin{gathered}
n_{v}^{ \pm}=\frac{1}{\pi} \operatorname{arcctg} \frac{\varepsilon_{a}+R \pm t}{\Gamma}, \quad n_{l}^{ \pm}=v_{l}^{ \pm} \cdot \Theta_{H}\left(E_{\mathrm{F}}-\omega_{l}^{ \pm}\right), \\
v_{l}^{ \pm}=\left(1+\frac{\Gamma}{\pi} \frac{E_{g}}{\left(E_{g} / 2\right)^{2}-\left(\omega_{l}^{ \pm}\right)}\right)^{-1}
\end{gathered}
$$

и значения $\omega_{l}^{ \pm}$определяются из уравнений $\omega-\varepsilon_{a} \mp t$ $-\Lambda(\omega)=0$ для энергий $|\omega|<E_{g} / 2$.
Повторно рассмотрим адсорбцию $\mathrm{Na}$ и $\mathrm{Cl}$ на $6 H-\mathrm{SiC}$ $p$ - и $n$-типов проводимости соответственно. В соответствии с данными работы [16] для $\sigma$-связей $s$-орбиталей атомов натрия $t=2.82$ эВ и $p$-орбиталей атомов хлора $t=4.74$ эВ. Расчет по формулам (9) дает для решетки атомов натрия, сформированной на $p-6 \mathrm{H}-\mathrm{SiC}$, значения $\widetilde{Z} \sim 0.7$ и 0.6 для С- и Si-грани соответственно, что существенно меньше значений $Z_{0} \sim 0.9$ и 1.0 для одиночного атома натрия, адсорбированного на тех же гранях. Для решетки атомов хлора, сформированной на $n-6 H-\mathrm{SiC}$, $\widetilde{Z}=-0.6$ и -0.5 для $\mathrm{C}-$ и $\mathrm{Si}-г$ рани соответственно, что меньше по модулю значений $Z_{0}=-0.8$ и -0.9 для одиночного атома хлора, адсорбированного на тех же гранях. Таким образом, в рассмотренных случаях прямой обмен приводит к существенной деполяризации адатомов.

Косвенный обмен представляет собой виртуальные межатомные переходы электронов через состояния подложки [7]. В отличие от дипольного взаимодействия, возникающего только в случае наличия заряда у адатомов, и прямого обмена, проявляюшегося лишь при $\Theta \rightarrow 1$, косвенный обмен присутствует всегда. Здесь мы не будем обращаться к общей теории, а рассмотрим адсорбированный „димер“, атомы которого связаны не прямым (как у реального дира), а косвенным обменом. Как показано в работе [17], функция Грина такого „димера““ определяется выражением (7) при замене энергии перескока $t$ на

$$
t_{\text {indir }}=\left|V^{2} \sum_{\mathbf{k}} \frac{\exp (i \mathbf{k r})}{\omega-\varepsilon_{\mathbf{k}}+i 0^{+}}\right|,
$$

где $\mathbf{k}$ и $\varepsilon_{\mathbf{k}}-$ волновой вектор и энергия электрона подложки, $|\mathbf{r}|=a_{0}$. Воспользовавшись оценками работы [17], положим $t_{\text {indir }} \approx 2 V^{2} / W$, где $W$ - ширина валентной зоны $\mathrm{SiC}$. Так как $\Gamma=\pi V^{2} \rho_{\text {sub }} \approx 4 \pi V^{2} / W$, получаем $t_{\text {indir }} \approx \Gamma / 2 \pi$. Тогда для адсорбции атомов натрия на $6 H$-SiC получаем $t_{\text {indir }} \approx 0.10 \quad$ (С-грань $)$

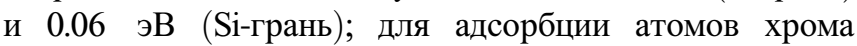

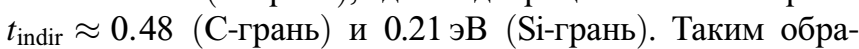
зом, $t_{\text {indir }} / t \ll 1$. Тем не менее ясно, что косвенное взаимодействие, как и прямое, приводит к деполяризации адатомов.

\section{4. Заключение}

В настоящей работе мы прибегали к многочисленным упрощениям, как при оценке параметров, так и при выборе моделей. Более того, каждый канал взаимодействия адатомов мы рассматривали как действующий независимо от других каналов. Такой подход позволил продемонстрировать, что дипольные силы являются доминирующей причиной деполяризации. Поэтому приведенные нами результаты можно считать лишь полуколичественными. Результаты эти, однако, могут быть достаточно просто (в принципе) проверены в эксперименте. Действительно, изменение работы выхода адсорбционной 
системы, даваемое выражением $\Delta \phi(\Theta)=-\Theta \Phi Z(\Theta)$, где $\Phi=4 \pi e^{2} d N_{\mathrm{ML}}$, однозначно связано с зарядом адатома. Таким образом, при $\Theta \ll 1$ значение $\partial \Delta \phi(\Theta) / \partial \Theta$ позволяет оценить заряд одиночного адатома $Z_{0}$, а величина $\Delta \phi(\Theta=1)$ дает информацию о заряде $Z_{\mathrm{ML}}$. Более того, отношение $Z_{0} / Z_{\mathrm{ML}}$ можно получить чисто опытным путем, не прибегая к дополнительным предположениям о величине расстояния $d$ между подложкой и адслоем. Далее, величина $\Delta \phi(\Theta)$ связана с изменением поверхностной проводимости подложки $\Delta \sigma(\Theta)$ соотношением $|\Delta \sigma(\Theta) / \Delta \phi(\Theta)|=\mu / 4 \pi d$, где $\mu$ - подвижность носителей, которая, как показывают оценки, слабо зависит от $\Theta$ (см. работу [18] и ссылки, приведенные там). Следовательно, для проверки полученных нами результатов вместо измерений $\Delta \phi(\Theta)$ можно измерять $\Delta G(\Theta)$. Полученные оценки представляют интерес также для физики барьера Шоттки: в работе [11] показано, что наличие экстремума у функции $\Delta \phi(\Theta)$ (при некотором значении $\Theta^{*}$ ) свидетельствует о пиннинге уровня Ферми на контакте металл-полупроводник.

\section{Конфликт интересов}

Авторы заявляют об отсутствии конфликта интересов.

\section{Список литературы}

[1] Silicon Carbide: recent major advances, ed. by W.J. Choyke, H. Matsunami, G. Pensl (Berlin-Heidelberg, Springer. 2004). E-address: http://www.springer.de

[2] Advances in Silicon Carbide. Processing and Applications, ed. by S.E. Saddow, A. Agarwal (Boston-London, Artech House, 2004). Eaddress: www.artechhouse.com

[3] A.A. Lebedev. Semicond. Sci. Technol., 21, R17 (2006).

[4] А.А. Лебедев, П.А. Иванов, М.Е. Левинштейн, Е.Н. Мохов, С.С. Нагалюк, А.Н. Анисимов, П.Г. Баранов. УФН, 189, 803 (2019).

[5] С.Ю. Давыдов, О.В. Посредник. ФТП, 54, 1197 (2020).

[6] С.Ю. Давыдов, О.В. Посредник. ФТП, 55, 326 (2021).

[7] О.М. Браун, В.К. Медведев. УФН, 157 (4), 631 (1989).

[8] С.Ю. Давыдов, А.В. Зубов. ФТТ, 62, 1302 (2020).

[9] Физические величины. Справочник, под ред. Е.С. Григорьева, Е.3. Мейлихова (М., Энергоатомиздат, 1991).

[10] Краткий справочник физико-химических величин, под ред. К.П. Мищенко и А.А. Равделя (Л., Химия, 1974).

[11] С.Ю. Давыдов, С.В. Трошин. ФТТ, 50, 398 (2008).

[12] С.Ю. Давыдов. ФТТ, 58, 1182 (2016).

[13] С.Ю. Давыдов. ФТТ, 58, 779 (2016).

[14] N.M.R. Peres, F. Guinea, A.H. Castro Neto. Phys. Rev. B, 73, 125411 (2006).

[15] С.Ю. Давыдов. ФТТ, 54, 2193 (2012).

[16] W.A. Harrison. Phys. Rev. B, 27, 3592 (1983).

[17] S. Alexander, P.W. Anderson. Phys. Rev., 133, A1594 (1963).

[18] С.Ю. Давыдов, О.В. Посредник. ЖТФ, 87, 635 (2017).

Редактор А.Н. Смирнов

\section{Electron states of atoms in monolayers adsorbed on silicon carbide}

\author{
S.Yu. Davydov ${ }^{1}$, O.V. Posrednik ${ }^{2}$ \\ ${ }^{1}$ loffe Institute, \\ 194021 St. Petersburg, Russia \\ ${ }^{2}$ St. Petersburg State Electrotechnical University (ETU), \\ 197376 St. Petersburg, Russia
}

\begin{abstract}
Estimates of charge transfer between adsorbate monolayer and $\mathrm{SiC}$ substrate based on account of the dipole-dipole repulsion and exchange interactions are presented. It is demonstrated that all the interaction channels lead to adlayer depolarization. Role of the band and local adatoms states are analyzed. Methods of

experimental verification of the obtained results are proposed.
\end{abstract}

